\title{
Diagnosis and Management of Deeper Neck Infections - A Review
}

\author{
Dr. Kumaran Alias Ramesh Colbert, M.S., Dnb ${ }^{1}$, Dr. S. Devakumari, M.D.S ${ }^{2}$ \\ ${ }^{I}$ (Associate Professor\& HOD, Department of Otorhinolaryngology, Indira Gandhi Medical College \& Research \\ Institute (Government of Puducherry Institution), PUDUCHERRY - 605 009, PONDICHERRY UNIVERSITY, \\ INDIA) \\ ${ }_{2}^{2}$ Assistant Professor, Department of Dentistry, Idira Gandhi Medical College \& Research Institute \\ (Government of Puducherry Institution), PUDUCHERRY - 605 009, \\ PONDICHERRY UNIVERSITY, INDIA)
}

\begin{abstract}
Deeper Neck Infections (DNI) of odontogenic origin are more common in adults than in children. The diagnosis of DNI is difficult due to lack of common clinical symptoms and signs. The diagnosis is further difficult due to deep seated infections which are covered by normal tissues of neck. Early treatment and intervention are important to reduce the morbidity and mortality. Proper antibiotic regimen and the duration of the treatment are important. Any impending airway obstruction should be closely monitored. Decision on treating the patient only with antibiotics or requirement of a surgical intervention depends on the progress of the patient and CT scan reports. Early surgical intervention is demanded due to proximity to important structures like Carotid Artery and Jugular Vein. DNI requires prompt treatment to prevent the most dreadful complications like necrotising fascitis, mediastinitis, carotid A erosion and jugular Vein thrombosis. DNI are not common in day-to-day practice, the knowledge of diagnosis, recognition of comorbid illness and treatment protocols are imperative to effectively treat these life threatening infections. Delay in diagnosis of DNI has high morbidity and mortality rates. This article reviews about the common clinical features of DNI and their management protocols summarizing recent clinical studies.
\end{abstract}

Keywords: Neck and head abscess, Retropharyngeal, Para pharyngeal, peritonsillar.

\section{Introduction}

Deeper Neck Infections (DNI) of head and neck have significant morbidity and mortality. Deep neck infections are odontogenic origin in adults while it is due to upper respiratory system infection in children. It is a challenge to treat these life threatening infections for both the physicians and the surgeons. Complications were reduced because of the modern microbiological tools, hematology, sophisticated imaging techniques (CT \& MRI), modern antibiotics, modern intensive care protocols and surgical techniques. Management of DNI is one of the most debated topic due to obscure clinical picture, need for sophisticated diagnostic tools and demand of early surgical intervention to prevent impending complications.

\section{Discussion}

DNI's are due to spread of infection from adenotonsillar tissue, odontogenic infections and salivary gland infections. The contiguous spread of infection from these head and neck spaces and fascial planes may be either as cellulitis or as abscess formation. ${ }^{[1,2]}$ It is further complicated by the presence of diabetic mellitus both in children and adults. In children, etiology is secondary to pharyngeal infections (Pharyngitis and tonsillitis) and in adults it is secondary to odontogenic infections. ${ }^{[3,4,5,6]}$ The DNI had decreased with modern antimicrobial therapy otherwise these infections lead to serious morbidity and mortality. Although DNI are not common in day-to-day practice, the knowledge of treatment protocol of DNI are important to promptly treat these life threatening complications.

\section{Etiology}

The causes Of DNI's are Tonsillar Infections, Dental Infections, Oral Surgical procedures, Salivary Gland infections, Trauma to Oral Cavity and Pharynx, Foreign Body aspiration, Cervical Lymphadenitis, Brachial Cleft Cysts. Thyroglossal duct Cysts, thyroiditis, mastoiditis and Bezold Abscess, Iv drug use, Necrosis ands Suppuration of malignant lymph nodes and idiopathic. ${ }^{[7]}$

\section{Comorbid Illness}

DNI are complicated by other comorbid illness. The most common being Diabetes Mellitus. LEE et al showed higher incidence of DM as comorbidities but it was not related to the major rate of complications. In his view the complication are more with Diabetes if more than two neck spaces were involved. ${ }^{[8]}$ HUANG et al in his study confirmed that patient with DNI's associated with Diabetes had longer duration of hospital stay and 
required intubations and tracheostomy than without $\mathrm{DM} \cdot{ }^{[9]}$ Other important comorbid illness to be considered were Immunosuppressed states like HIV, patients on chemotherapy or Patients on Immunosuppressive drugs for transplantation. These patients develop infection with atypical organisms and more complications. ${ }^{[7]}$

\section{Pathophysiology And Spread Of Infection}

The layers of the cervical fascia create potential deep neck spaces in the head and neck region which are responsible for the spread of infections. Deep neck spaces are generally divided as 1 . in the face: buccal, canine , masticator and parotid spaces. 2.suprahyoid:peritonsillar,submandibular, sublingual and lateral pharyngeal spaces 3:infrahyoid:anterior, visceral 4.extending in to the length of the neck: retropharyngeal, danger space, prevertebral and carotid sheath spaces. ${ }^{[10]}$ The spaces of neck have a complex anatomy, and the precise location of infection is difficult. Further they are deeply located and not amenable to palpation or visualization. Deep neck spaces are covered by near normal tissues and they are adjacent to important structures like Carotid Artery, Jugular Vein and Vertebrae. Sloughing of tissues, neural Dysfunction, vascular erosions, necrotizing fascitis, osteomyelitis are potential complication of DNI. The surgical access to these potential spaces is complicated by proximity to Neurovascular structures and the morbidity caused to the superficial structures to gain access. Surgical Drainage of these spaces are complex because they communicate each other and other parts of body (mediastinum and coccyx) ${ }^{[7]}$

The spread of infection from dental infections is through the lymph nodes. The lymph nodes undergo suppuration and focal abscess formation and they spread from one space to another early. Once infection is set, it spreads as cellulitis or abscess. The clinical signs and symptoms are due to pressure effect on important structures due to the spread of infection or the direct involvement of important structures.

For Example: An untreated Tonsillar abscess or pericoronitis spreads in to lateral Pharyngeal space from there they spread to Retropharyngeal space and prevertebral space and then to the chest leading to Mediastinits. An alternative path from lateral pharyngeal space is to the contents of Carotid sheath. Lateral pharyngeal space infection can per se cause airway obstruction at the level of pharynx. The mean progression of DNI is 8.51 days. Necrotising fascitis is the dreadful complication of DNI.

\section{Microbiology}

The microbiology of DNI are mixed aerobic and anaerobic organisms predominant of oral flora. Both gram positive and gram negative organisms were cultured. ${ }^{[11,12,13]}$ Anaerobes were found in almost $50 \%$ of the cases. The predominant anaerobes were Pepto Streptococcus Species, prevotella, Porphyromonas and Fusobacterium necrosum. Group A and Group B Streptococcus SP, Staphylococcus aureus and Hemophilus were common aerobic organisms. Wang et al ${ }^{[13]}$ isolated klebsiella pneumoniae in patients with DM. Organisms are more virulent and more than two-thirds were betalactamase producers. There are increasing numbers of infections sustained by MRSA in children ${ }^{[14,15,16]}$ Patients who are already on antibiotics the isolates were be betalactamase producing strains ${ }^{[17]}$

\section{Clinical Features}

It is important to elicit recent dental procedures, URTI, trauma, relevant medical history, onset, duration and antibiotics prescribed, immunosuppressive drug intake, steroids, chemotherapy and H/O HIV and TB.

\section{Signs And Symptoms}

Fever, Pain, Dysphagia, Dyspnea, Tenderness and Swelling in the neck.

\section{Common Presentation}

Medial Displacement of Lateral Pharyngeal wall and tonsil - Para pharyngeal space infection. Trismus - Masticator space infection. Swelling in the angle of the mandible without Trismus -odontogenic parotid space infection. Torticollis and decreased neck movement- Prevertebral space infection. Hoarseness of voice- true vocal cord paralysis. Horner's Syndrome- Involvement of Cervical Sympathetic chain. Fever SpikesSepticaemia, internal Jugular Vein Thrombo Phlebitis. Tachynea and breathlessness- Impending airway obstruction. Crepitus in soft tissue- Necrotising fascitis.

\section{Investigations}

Plain radiographs-lateral cervical films are quicker and show retropharyngeal soft tissue widening and gas shadow suggesting of abscess especially in children.

CT is usually ordered when DNI are presented at level II, III and IV. CT aids in diagnosis as well as surgical planning. ${ }^{[18,19]}$ Contrast enhanced CT ( Coronal and axial Planes) is preferred. CT is highly sensitive for DNI (91\%) . DNI appears as low density image on CT. Contrast enhancement of abscess wall, the relationship to adjacent fascial planes and soft tissue oedema can be elicited. ${ }^{[19,20]}$ 
Ultrasound is a cost effective tool. Ultrasound can differentiate between abscess and cellulites ${ }^{[18][19]}$. It is not reliable due to the difficulty in diagnosing Deep Seated Infections. Ultrasound can guide in aspiration. MRI gives excellent soft tissue imaging and can identify internal Jugular Vein thrombosis. ${ }^{[19.20]}$

Laboratory Investigations

- Complete blood cell count with differential count.

- Culture and sensitivity via aspiration.

- $\quad$ reactive protein and ESR can be monitored as predictors of clinical response. ${ }^{[19]}$

\section{Airway Maintenance}

Patients with DNI should be carefully monitored for airway obstruction. Maintenance of airway becomes difficult due to inflammation in airway, fragility of tissues, tissue rigidity, trismus. ${ }^{[21]}$ and the risk of foreign Body aspiration. The need for tracheostomy/ intubation should be carefully assessed and promptly done to prevent further damage to already inflamed tissues. Ovassapian et al suggested intubation anaesthesia as the first choice ${ }^{[21]}$. If airway is complicated by oedema and distortion or if the intubation had failed then tracheostomy had to be considered. The current literature states the need for Tracheostomy is $12-16 \%{ }^{[19]}$.

\section{Antibiotic Policy}

All odontogenic infections and pharyngeal infections entering into spaces in face and neck should be started with empirical antibiotics with coverage of gram positive, gram negative aerobes and anaerobes. The inclusion of compound active against beta lactamase producing organisms should be considered. However aspirated specimen from the infective focus should be sent as early as possible for aerobic and anaerobic culture and $\mathrm{AFB}$ ( acid fast bacilli staining if tuberculosis suspected). Alteration in antibiotic regimen can be done with culture and sensitivity report. There are no universal agreement on empirical antibiotic therapy. The preferred choices are Amoxycillin+clavulanic acid+ Metronidazole or Ceftriaxone+ Metronidazole.

\section{Surgical Drainage}

Surgical Drainage is done using wider neck incisions. GA for drainage is difficult and can be done under LA and sedation as far as possible. Wider neck incisions are made based on the extent of infection and involvement of neck spaces based on CT. Layer wise dissection is indicated with due precaution to the adjacent neurovascular structures. Wider incision and dissection drains most of the pus and further drainage is done with a sinus forceps and sometimes by introducing little finger into the anticipated space. After adequate drainage incision is closed for half a length in layers and corrugated rubber drain is secured. The drain is removed after 24-48hrs. Further management other than cervical drainage ranges from Debridement, Thoracotomy, Thoracic drainage and mastoidectomy depending on the clinical situation. ${ }^{[22]}$

\section{Conclusion}

1. The cause of DNI in children are due to Tonsillar infection and in adult the etiology is odontogenic

2. The most common space infection that leads to DNI are submandibular and parapharyngeal spaces.

3. Infections are polymicrobial comprising of oropharyngeal flora predominantly.

4. CT is the diagnostic aid of choice to determine the level of infection and to aid in surgical planning.

5. Empirical intravenous antibiotics should be promptly started covering gram positive, gram negative aerobic and anaerobic pathogens.

6. Airway Obstruction should be monitored and if obstruction is seen decision is made on fibero optic intubation or tracheostomy.

7. Surgical Drainage is done as early as possible with wider neck incisions. Debridement is indicated in case of necrotising fascitis.

\section{References}

[1]. Sakagushi M, Sato S, Ishiyama T, Katsuno S, Tagushi K. Characterization and management of deep neck infection.

[2]. J Oral Maxillofac Surg. 1997, 26:131-134.

[3]. Osborn TM, Assael LA, Bell RB. Deep Space Neck Infection: Principles of Surgical Management. Oral Maxillofacial Surg Clin N Am. 2008, 20:353-365.

[4]. Conrad DE, Parikh SR. Deep Neck Infections. Infect Disord Drug Targets. Feb 17 2012; [Medline].

[5]. Chang L, Chi H, Chiu NC, Huang FY, Lee KS. Deep neck infections in different age groups of children. J Microbial Immunol Infect. Feb 2010;43(1):47-52. [Medline].

[6]. Wang LF, Tai CF, Kuo WR, Chien CY. Predisposing factors of complicated deep neck infections: 12 -year experience at a single institution. J Otolaryngol Head Neck Surg. Aug 2010;39(4):335-41. [Medline].

[7]. Poeschl PW, Spusta L, Russ Mueller G, Seemann R, Hirschl A, Poeschl E, et al. Antibiotic susceptibility and resistance of the odontogenic microbiological spectrum and its clinical impact on severe deep space head and neck infections. Oral Surge Oral Med 
Oral Pathol Oral Radiol Endod. Aug 2010;110(2):151-6. [Medline]. Alan D Murray et al, emedicine.medscape.com/article/837048overview

[8]. Lee JK, Kim HD, Lim SC. Predisposing Factors of Complicated Deep Neck Infection: An Analysis of 158 cases. Yonsei Med J. 2007, 48(1):55-62.

[9]. Huang TT, Tseng FY, Liu TC, Hsu CJ, Chen YS. Deepneck infection in diabetic patients: comparison of clinical picture and outcomes with nondiabetic patients. Otolaryngol Head Neck Surg. 2005, 132(6):943-7

[10]. Brook I, Microbiology and management of peritonsillar, retropharyngeal, and parapharyngeal abscesses, J Oral Maxillofac Surg, 2004;62:1545-50.

[11]. Huang TT, Liu TC, Chen PR, et al., Deep neck infection: analysis of 185 cases, Head Neck, 2004;26:854-60.

[12]. Bakir S, Tanriverdi H, Gün R, et al., Deep neck space infections: a retrospective review of 173 cases, Am J Otolaryngol, 2012;33:5663.

[13]. Wang LF, Kuo WR, Tsai SM, Huang KJ, Characterizations of life-threatening deep cervical space infections: a review of one hundred ninety-six cases, Am J Otolaryngol,2003;24:111-7.

[14]. Duggal p,Nasseri,sobol SE,The increased risk of methicillin resistant staphylococcus neck infections in young children,Laryngoscope, 2011;121:51-5

[15]. Cabrera CE, Deutsch ES, Eppes S, et al., Increased incidence of head and neck abscesses in children, Otolaryngol Head Neck Surg, 2007;136:176-81.

[16]. Ossowski K, Chun RH, Suskind D, Baroody FM, Increased isolation of methicillin-resistant Staphylococcus aureus inpediatric head and neck abscesses, Arch Otolaryngol Head Neck Surg, 2006;132:1176-81

[17]. Coticchia JM, Getnick GS, Yun RD, Arnold JE, Age-, site-, and time-specific differences in pediatric deep neck abscesses,Arch Otolaryngol Head Neck Surg, 2004;130:201-7.

[18]. Silvia Garazzino et al,Deep neck infections-Review of their clinical presentation, diagnosis and management,European infectious Disease2012;6(2);82-6.

[19]. Coelho MS et al , Deep neck infections-classification in levels of severity,Intl. Arch. Otorhinolaryngol.,sao Paulo,2009,v.13,n.2,p.184-188.

[20]. smith JL 2 nd, Hsu JM,Chang J, predicting deep neck space abscess using computed tomography, Am J Otolaryngol,2006;27:244-7

[21]. 11. Ovassapian A, Tuncbilek M, Weitzel EK, Joshi CW. AirwayManagement in Adult Patients with Deep Neck Infections:A Case Series and Review of the Literature Anesth Analg. 2005, 100:585-9.

[22]. Alexandre Babá Suehara et al, Deep neck infection - analysis of 80 cases, Rev Bras Otorrinolaringol 2008;74(2):253-9.

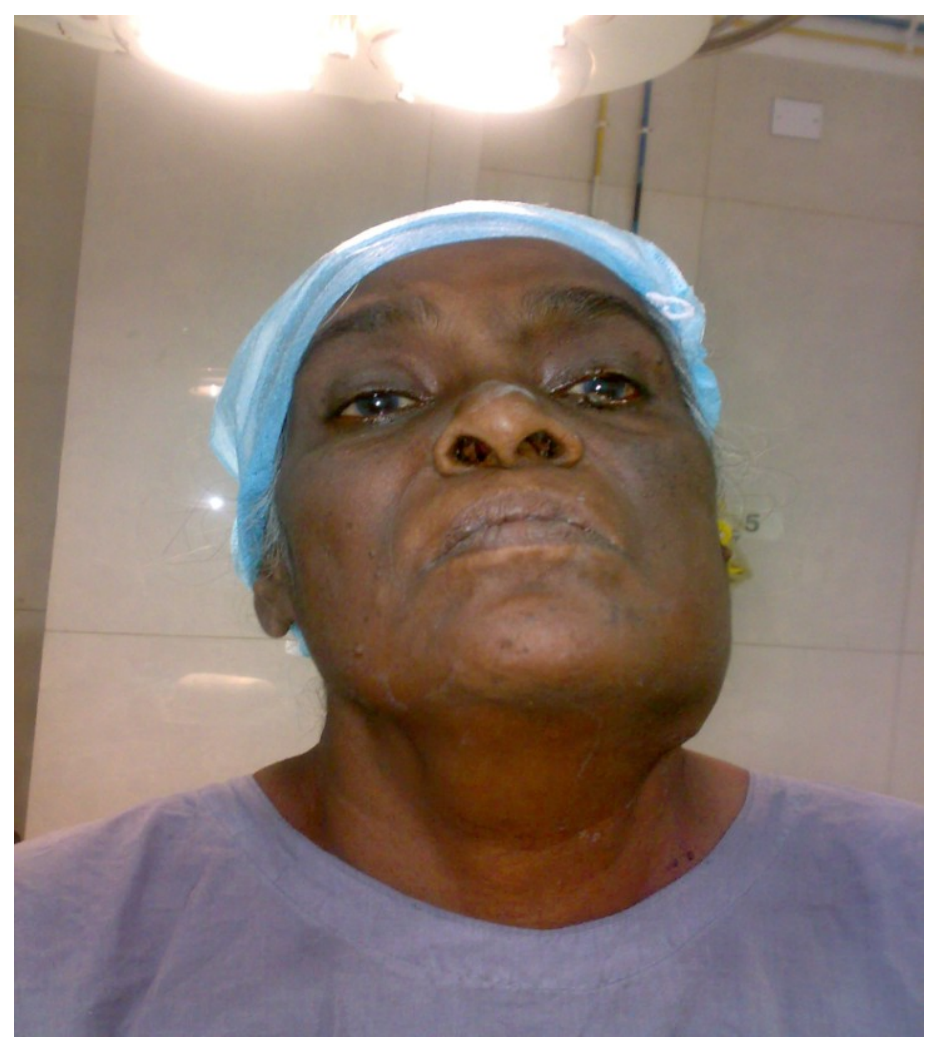

Figure 1-Case-1-Parapharyngeal space infection 


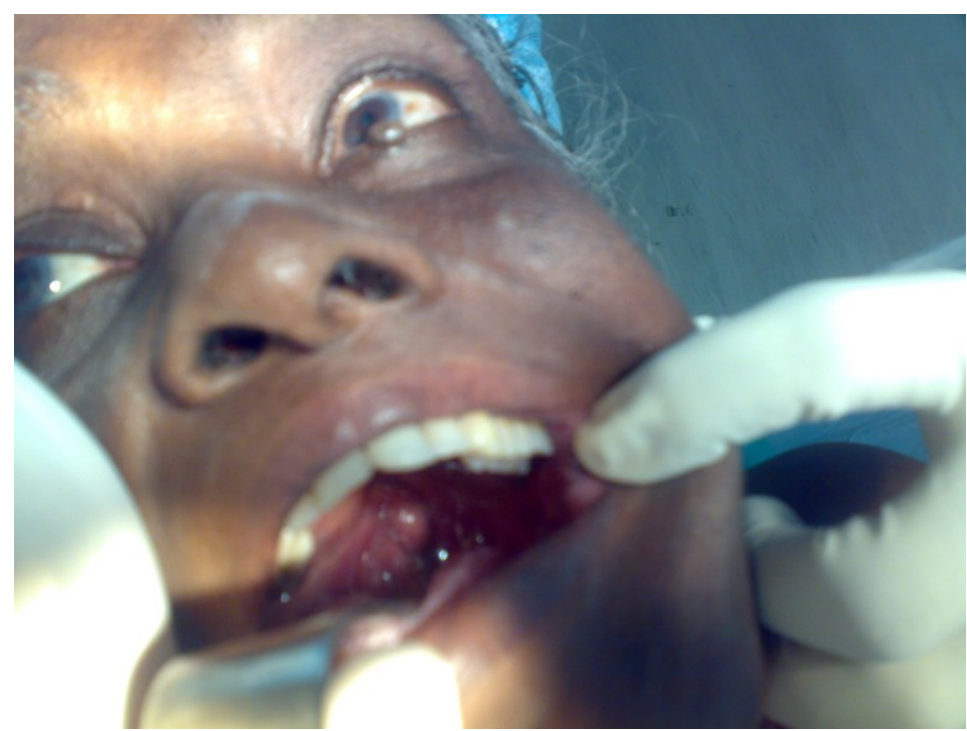

Figure 2-Case-1-Swelling in left pharyngeal wall

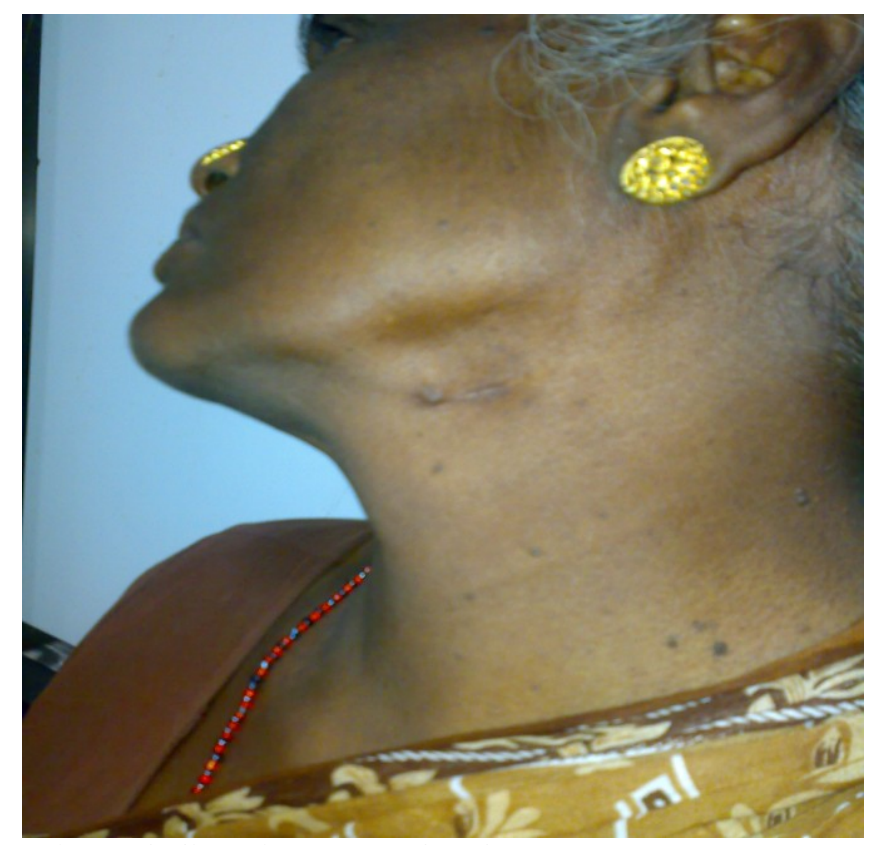

Figure 3-Case-1-post op after 3 months

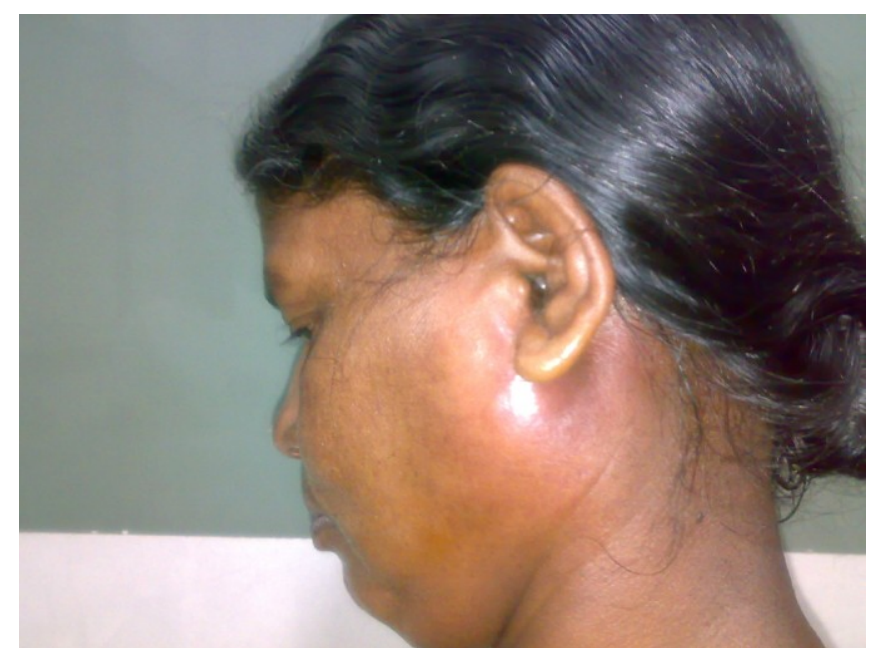

Figure 4-Case-2-Odontogenic parotid space infection due to abscess 


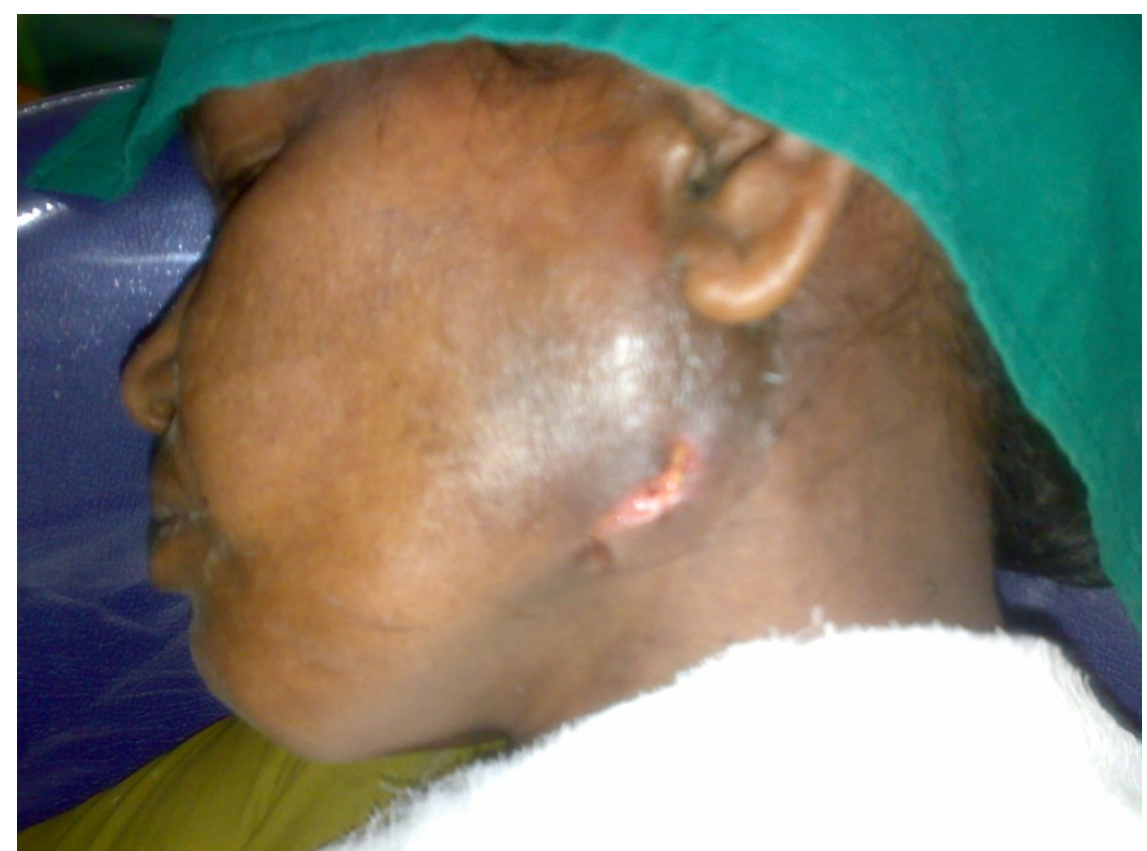

Figure 5-Case -2 -Healing after seven days

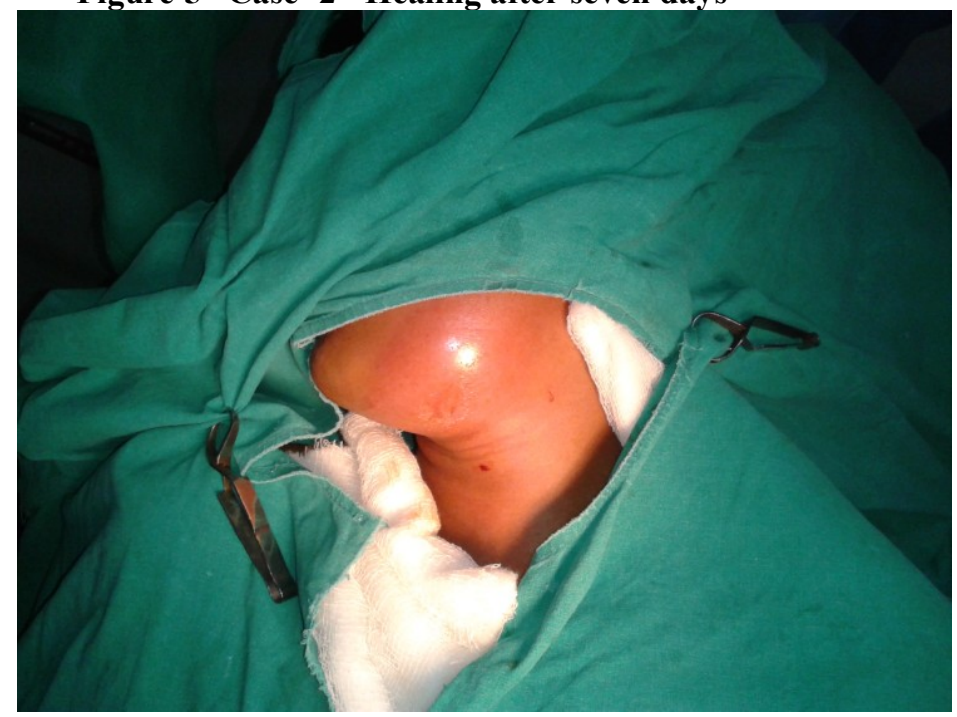

Figure 6 case 3 SUBMANDIBULAR ABSCESS

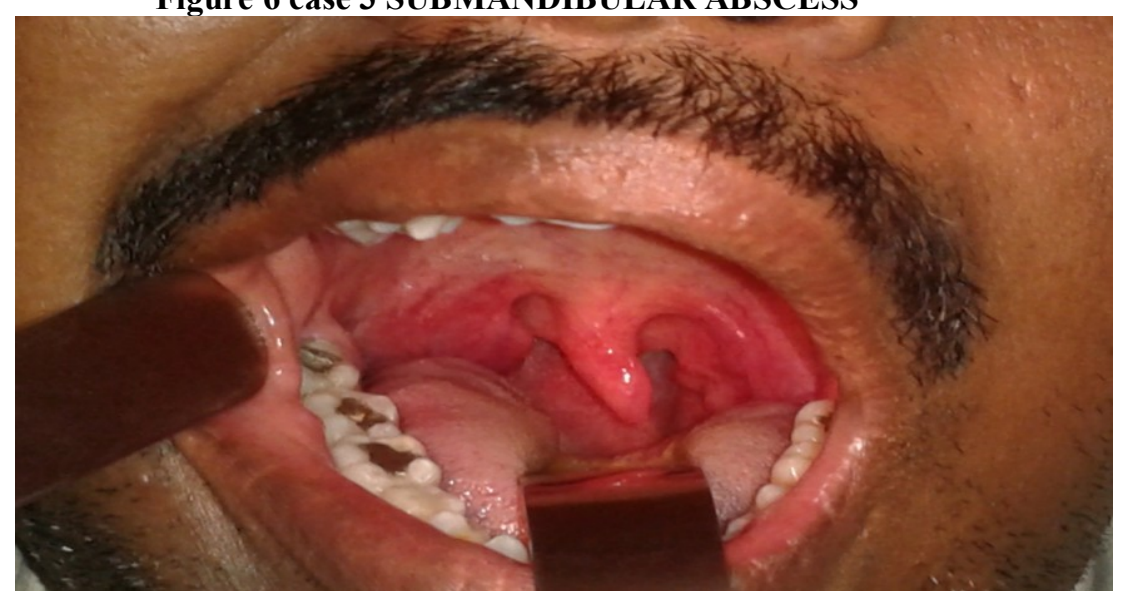

Figure 7 CASE 4 RIGHT PERITONSILLAR ABSCESS 